\title{
Génesis Semiótica de los Enteros
}

\author{
Semiotic Genesis of Integers
}

François Pluvinage $^{*}$
Patricia Flores $^{* *}$

\begin{abstract}
Resumen
La presentación tradicional del anillo de los enteros se enfrenta a dificultades de comprensión del producto por parte de los alumnos, con la famosa regla de los signos. Nuestra hipótesis de trabajo es que la sola representación del producto de dos naturales como área de un rectángulo puede dificultar la visión del producto de dos enteros, y que entonces, en el esquema de los Espacios de Trabajo Matemático, se necesita otro soporte visual para la génesis semiótica de los enteros. Experimentamos un recurso didáctico vinculado con una construcción publicada por Descartes en su suplemento geométrico del Discurso del Método. Observamos que este acercamiento geométrico del producto puede generar dificultades, en particular relacionadas con la propiedad distributiva del producto sobre la suma. Una explicación resulta de la consideración del producto de enteros como procept. De donde surge la propuesta de tomar en cuenta la homotecia para la elaboración de una progresión didáctica al servicio de la enseñanza de los enteros y más generalmente de los números con signo.
\end{abstract}

Palabras clave: Producto de Números. Regla de los Signos. Distributividad. Geometría del Plano. Espacio de Trabajo Matemático. Génesis Semiótica. Homotecia.

\begin{abstract}
The traditional presentation of the integers ring generates difficulties for learning the product with its wellknown signs rule. Our working hypothesis is that the solely representation of the product of two natural numbers by the area of a rectangle can hinder the vision of the product of two integers. Referring us to the scheme of Mathematical Working Spaces, we need another representation for the semiotic genesis of the integers. We tested didactical resources coming out from a geometrical construction made by Descartes in his geometric supplement of the Discourse on the Method, but we observed that this geometric introduction of the product can also produce difficulties, because it does not easily illustrate the distributive law. We may explain this phenomenon considering the product of numbers as a procept. Thus, the homothetic transformations of the Cartesian plane constitute a convenient geometrical resource that we may propose for teaching of the integers and, more generally, the numbers with a sign.
\end{abstract}

Keywords: Multiplication of Numbers. Signs Rule. Distributive Law. 2D Geometry. Mathematical Working Spaces. Semiotic Genesis. Dilation.

\footnotetext{
"Docteur es Sciences, Université Louis Pasteur (ULP) de Strasbourg; Investigador titular, Departamento de Matemática Educativa, Centro de Investigación y de Estudios Avanzados del Instituto Politécnico Nacional (DME, Cinvestav-IPN), Av. Instituto Politécnico Nacional 2508, Col. San Pedro Zacatenco, C.P. 07360, México, D.F. ; fpluvinage@cinvestav.mx

${ }^{* *}$ Doctora en Ciencias especialidad Matemática Educativa, Centro de Investigación y de Estudios Avanzados del Instituto Politécnico Nacional ; Profesora e Investigadora, Universidad Pedagógica Nacional (UPN), Carretera al Ajusco No. 24 Col. Héroes de Padierna Del. Tlalpan, C.P. 14200, México, D.F. ; pflores63@ hotmail.com
} 


\section{Introducción}

Las dificultades señaladas tanto operativas como conceptuales que tienen los alumnos en la adquisición de las estructuras numéricas $\mathbb{Z}, \mathbb{Q}, \mathbb{R}$ son producto de los obstáculos vinculados con la comprensión de los números negativos, que han sido descritos detalladamente desde hace mucho tiempo, en particular por Glaeser (1981) y Cid (2000). Estas dificultades influyen en el tratamiento de ecuaciones con radicales y en estudios de funciones reales.

Hoy existe un corpus de estudios didácticos sobre la enseñanza de los números negativos, sin embargo el conocimiento del mismo por la comunidad docente es insuficiente. Además no se observa una influencia notable de estos estudios didácticos en los programas curriculares y libros de texto actuales, que siguen ofreciendo espacios demasiado restringidos a los enteros y más generalmente los números negativos. En particular, hay una carencia general en la justificación del producto de dos números con signos: la regla de los signos, y una ausencia de representación sensible de este producto.

Nuestro estudio se enfoca sobre perspectivas de reforzamiento del aprendizaje de los números con signos, apoyadas sobre la teoría de los registros de representación y el esquema de los Espacios de Trabajo Matemático. El plan del artículo es el siguiente:

- Un primer apartado se dedica a consideraciones socioepistemológicas, a propósito de la aparición de los números negativos en la historia, en paralelo con las escrituras algebraicas.

- Luego se considera la enseñanza de los enteros, poniendo especial énfasis en la propiedad distributiva del producto sobre la suma.

- Estudiamos una visualización del producto de enteros y números con signo, fundamentada en una construcción de Descartes, y presentamos un estudio empírico que explota esta presentación.

- Presentamos en conclusión las perspectivas abiertas por el recurso geométrico de la homotecia. 


\section{Consideraciones socioepistemológicas}

\subsection{La introducción de los signos de operaciones numéricas}

¿Cuándo se introdujeron las notaciones actuales de las operaciones numéricas, de uso universal y presentes en las teclas de la calculadora más sencilla? Con respecto a esta pregunta, la enciclopedia en línea Wikipedia en inglés en el artículo Plus and minus signs (consultado en noviembre de 2015) da la respuesta: "Though the signs now seem as familiar as the alphabet or the Hindu-Arabic numerals, they are not of great antiquity”. Esta aparición la sitúa Cajori (1928, vol. 1, p. 128) en el fin del siglo XV: “The + and - symbols first appeared in print in Mercantile Arithmetic or Behende und hüpsche Rechenung auff allen Kauffmanschafft, by Johannes Widmann (born c. 1460), published in Leipzig in 1489. However, they referred not to addition or subtraction or to positive or negative numbers, but to surpluses and deficits in business problems." Sin embargo, en una página del libro de Widmann (Figura 1) aparece que se hacen operaciones aritméticas en el contexto mercantil considerado (donde aparecen quintal y libra, en alemán Zentner y $l b$ ). En efecto, en las líneas 5 y 6 desde abajo del párrafo, se ven los verbos que subrayamos: addieren (sumar en alemán) y susstrahieren (restar en alemán de la época), y se hace la resta 4539 - $387=4152$.

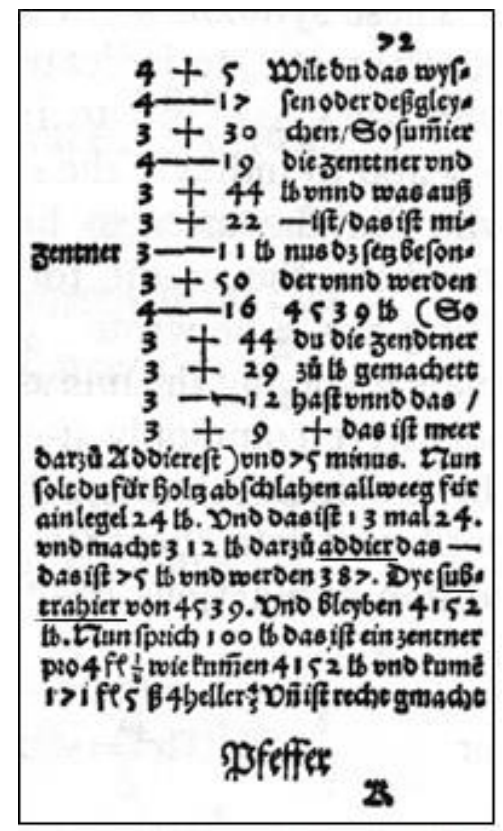

Figura 1 -Una página del libro de Johannes Widmann.

La fecha de publicación del libro (1489) es de tres años anterior al desembarque de Cristobal Colón en América (1492), que buscaba llegar a las Indias, una época de fuerte 
desarrollo del comercio. Otro factor de importancia fue la invención de la imprenta (Gutenberg, alrededor de 1449). En una conferencia titulada La Innovación y lo Digital impartida el 28 de enero del $2013^{1}$, el filósofo de las ciencias Michel Serres declara: “ $L a$ revolución digital en curso tendrá efectos al menos tan notables como en su tiempo los de la invención de la escritura, y luego la invención de la imprenta” (traducido del Francés). ¿Qué efecto tuvo la imprenta, precisamente en el caso que nos interesa aquí: los símbolos del cálculo aritmético? Nuestra hipótesis es que esta técnica necesitaba una normalización, que tomó la forma de fuentes de caracteres. La composición de un libro impreso no autorizaba la libertad de la escritura de un manuscrito y su copia, pero la obra obtenida se podía difundir a larga escala.

El signo de igualdad apareció por primera vez en el libro The Whetstone of Witte (el Hito de la Sabiduría) de Robert Recorde, publicado el año 1557. El autor comenta su elección de dos segmentos paralelos de igual longitud para representar el símbolo de igualdad, declarando (véase Figura 2): "bicause noe 2 thynges can be moare equalle" (porque dos cosas no pueden ser más iguales).

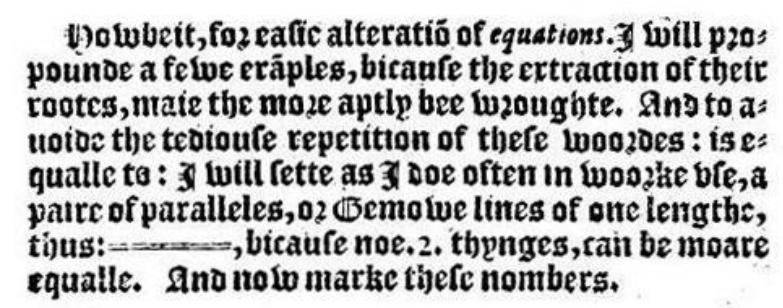

Figura 2 - Imagen tomada del libro de Recorde, publicada en el artículo de Wikipedia en Inglés Robert Recorde.

\subsection{Dos casi gemelos: el conjunto de los números negativos y el álgebra}

Una vez difundidas las notaciones de las operaciones numéricas, no pasó mucho tiempo, hasta que aparecieron dos avances matemáticos que todavía hoy tienen validez: los números negativos y el álgebra. Los negativos que nos interesan aquí son los que con los positivos determinan una estructura de anillo o de campo. Esto significa que se deben definir sobre ellos las operaciones a la vez aditivas y multiplicativas. Sin esta restricción, las cuentas con valores negativos ya se usaban en China antigua (con una convención todavía valida en contaduría: color negro para indicar ganancias y color rojo para indicar pérdidas o deudas). El

\footnotetext{
${ }^{1}$ Disponible en: https://www.canalu.tv/video/universite_paris_1_pantheon_sorbonne/michel_serres_1_innovation_et_le_numerique.11491
} 
álgebra que consideramos aquí es el tópico que los matemáticos llaman álgebra elemental y que trata de formación y resolución de ecuaciones reales.

Las obras de dos autores se destacan con respecto a estos tópicos: Simon Stevin (15481620) y François Viète (1540-1603). Algo que merece la atención es que ambos no sólo se dedicaron a estudios de matemáticas, sino también fueron hombres metidos en la vida pública. Stevin sostuvo una actividad importante como ingeniero, incluso militar, y Viète fue perito en derecho y administración. A Stevin se le atribuye el esbozo de la escritura decimal que conocemos hoy en su forma acabada con la coma o el punto decimal (por ejemplo 3,12 o 3.12 como escritura de 78/25), y no se podría olvidar que la contribución de este autor a la consideración de la recta numérica fue decisiva.

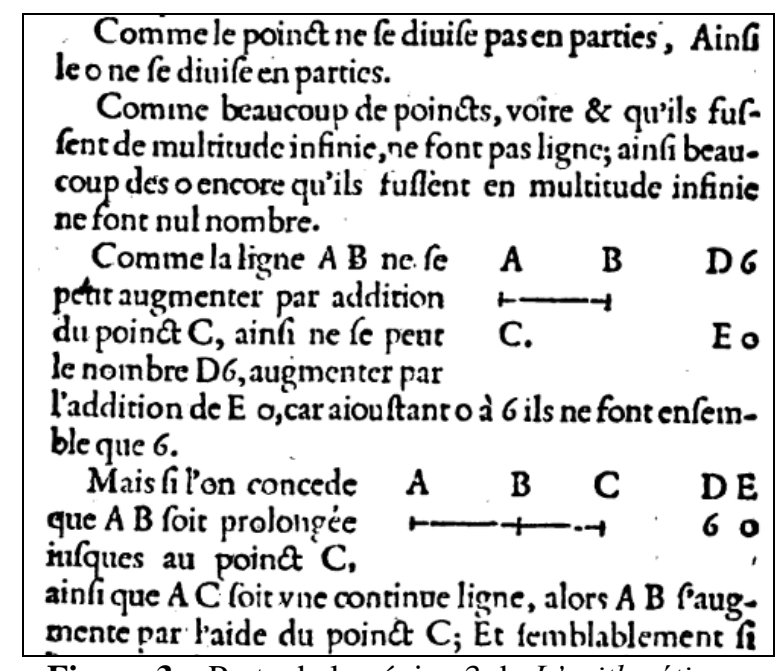

Figura 3 - Parte de la página 3 de L'arithmétique.

Algunas veces en la literatura científica se pretende que fue John Wallis (siglo XVII) el matemático que reconoció el vínculo entre números negativos y dirección, e introdujo la idea de la recta numérica como representación del sistema de los números. Esto no es exacto: La figura 3, tomada del libro L'arithmétique de Stevin (1585), pone de manifiesto una visión clara cuanto menos de la semirrecta numérica como representación de los números positivos. Stevin formula dos principios: a) La unidad es un número por ser divisible en partes, lo que es contrario a la idea expresada por Diofanto de Alejandría de que 1 es indivisible; b) El 0 no se divide en partes (Figura 3 arriba).

Más adelante en el mismo libro de Stevin (ibid. p. 166) aparecen los números positivos y negativos de manera explícita. La figura 4 reproduce una parte de la página 166 en la que la regla de los signos se presenta como teorema: Más multiplicado por más da producto más, menos multiplicado por menos da producto más, más multiplicado por menos o menos multiplicado por más da producto menos. Stevin no usa las variables, introducidas siete años 
después por François Viète (1591), sino propone un ejemplo numérico, considerándolo como genérico, el producto $(8-5) \times(9-7)$.

La presentación de la operación como un producto usual de dos enteros (a la izquierda del párrafo "Explication du requis") se apoya sobre la distributividad: El primer renglón -56 + 35 en la operación es el producto de $(8-5)$ por el factor -7. Esto pone de manifiesto que Stevin acepta la consideración de negativos aislados, aunque está en la lista de los obstáculos establecida por Glaeser (1981). En el cálculo meramente numérico de Stevin no se presentan magnitudes. Sin embargo, la representación geométrica de productos por rectángulos, presente en varias páginas del libro de Stevin, conduce a la consideración cuanto menos implícita, de magnitudes.

THE OREME.

1 lus multiplié par plus, donner produict plus,

duict plus, or pless multiplié par moins,ou moins multiplié par plus, donner produict moins.

Explication du donné. Soit 8-s multiplié parg-7,en cefte forte; $\rightarrow 7$ fois -5 , font +35 ( +35 , par ce que comme dict le theoreme, - par -, faict +) Puis - 7 fois 8 , faict - $66(-s 6$, par ce que commediat eft au theoreme, - par +, faic-) Et femblablement foit 8 - 5 , multiplié par le 9, \& donneront produicts 72 -45; Puis aiouftez $+72+35$ font 107. Puis aiouftez les - $56-45$, font - ior; Et foubftraict le 10I de 107 refte 6 , pour produia de telle multiplicatiō. De laquelle la difpoficion des characteres de l'operation eft telle: Explication du requis. Il faut de-

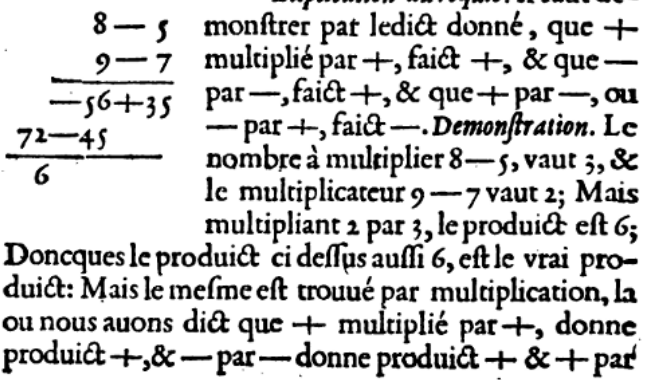

Figura 4 - Parte de la página 166 de L'arithmétique.

El problema de dimensiones se presenta de manera explícita en el libro de Viète, In Artem Analyticem Isagoge (VIÈTE, 1591) considerado como la obra en la que se introdujo el Algebra. En particular, Viète presenta de manera explícita operaciones que transforman una ecuación en otra equivalente (véase VIÈTE, 1591, proposiciones I a III, p. 7-8). En la proposición II en página 7 del libro (véase figura 5), se ve una simplificación por un factor A; hoy escribiríamos

$$
\mathrm{A}^{3}+\mathrm{BA}^{2}=\mathrm{ZA} \Leftrightarrow \mathrm{A}^{2}+\mathrm{BA}=\mathrm{Z}
$$


Viète considera las letras como representantes de magnitudes por naturaleza esencialmente positivas (no es seguidor de Stevin sobre aritmética). Por esta razón atribuye a $\mathrm{Z}$ en su texto la dimensión de una superficie (escribe: "Z plano") y no hace caso de la posibilidad $\mathrm{A}=0$. Además el lector observará la diferencia entre el texto de Viète y la escritura algebraica actual.

\section{Proponatur A Cubus, plùs $B$ in A Quadratum aquari $Z$ plano in A, Dicoper hypobitiafniu A Quadratum,plus $B$ in $A$ æquari $Z$ plano.}

Figura 5 - Texto en la página 7 de In Artem Analyticem Isagoge (VIÈTE, 1591)

\section{Los números negativos en el discurso matemático escolar}

Aspectos comunes de la introducción de números negativos en los programas curriculares y los libros de textos son la división del tema en trozos separados y el espacio reducido de la geometría en relación con los números negativos y el álgebra. En la guía para el maestro del programa oficial de estudio 2011 publicado por la SEP (SEP, 2011), se ve como aprendizaje esperado al fin del primer año de la secundaria (grado 7 de la escolaridad obligatoria): Soluciona problemas aditivos que conllevan el uso de números enteros, fraccionarios o decimales positivos y negativos. Los problemas multiplicativos pertenecen a un nivel más avanzado. Los estándares (SEP, 2011, p. 15) separan los tratamientos aditivos y multiplicativos:

1.2.1 Soluciona problemas aditivos que obligan a efectuar cálculos con expresiones algebraicas.

1.3.1. Resuelve problemas multiplicativos con expresiones algebraicas a excepción de la división entre polinomios.

Y en el eje temático "forma, espacio y medida", sólo un estándar se relaciona con cálculos posiblemente algebraicos:

2.2.1. Calcula cualquiera de las variables que intervienen en las fórmulas de perímetro, área y volumen.

Los libros de texto ponen énfasis sobre la estructura aditiva, presentando desplazamientos sobre la recta graduada y diversas situaciones isomorfas (crédito/debito, ascensores, nivel de agua en un tinaco, juego de fútbol americano, etc.). También, investigaciones sobre los negativos como la de Bruno y Martinón (1994) se enfocan en la estructura aditiva. En los libros de texto de nivel secundaria en matemáticas aprobados por la 
SEP en México el universo de situaciones que dan sentido a la estructura multiplicativa incluyendo los negativos es muy pobre. Lo más frecuente que se presenta en los libros es la distributividad del producto sobre la suma de números positivos ilustrada por la figura de dos rectángulos (Figura 6).

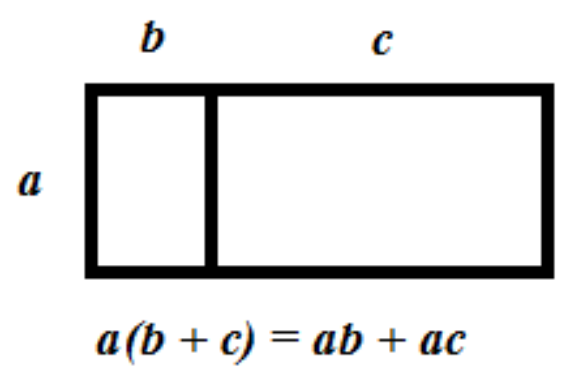

Figura 6 - Distributividad

No se expresan comentarios sobre el problema de dimensión generado por esta situación: Los factores son longitudes mientras los productos son áreas. Además la figura difícilmente se podría adaptar a la presencia de números negativos. Por eso, después de esta presentación, generalmente los libros "olvidan" esta interpretación y sólo conservan la distributividad, sea de manera directa, sea mediante una progresión del tipo $3 \times 4=12,3 \times 3=9$, $3 \times 2=6,3 \times 1=3,3 \times 0=0,3 \times(-1)=-3$, etc. para llegar a la "regla de los signos". El estudio detallado de Maz y Rico (2007) constata esta visión de los contenidos de los libros de texto en el contexto español.

Entre los libros de texto que consultamos están los libros para la telesecundaria producidos por la SEP que nos llamaron la atención por el cuidado que los autores tomaron en el diseño de las actividades propuestas a los estudiantes. Con respecto a nuestro tema, los libros (dos volúmenes) del grado 3 (SEP, 2009) que corresponden al último año de enseñanza básica. 


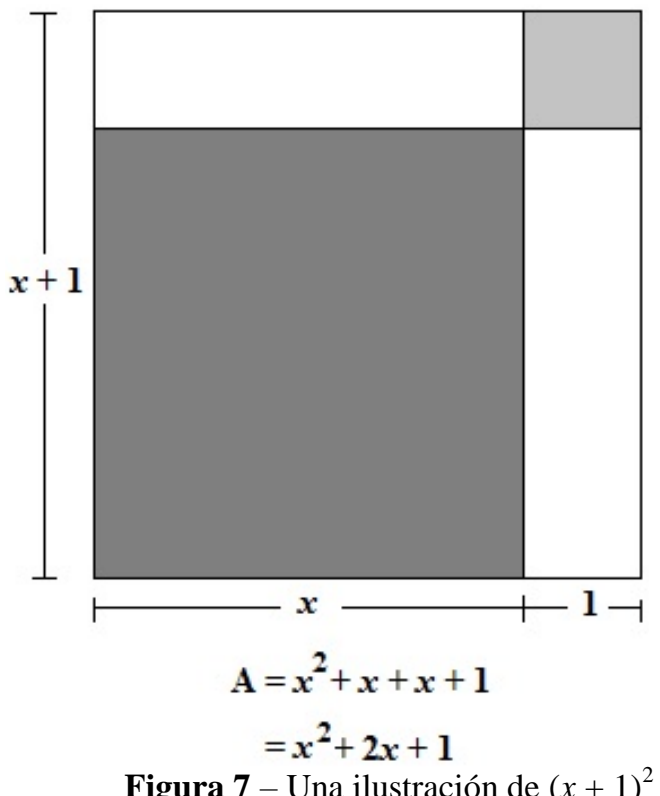

La primera figura que se encuentra en el volumen 1, p. 12, representa la igualdad $(x+1)^{2}=x^{2}+2 x+1$ (Figura 7). Esta figura viene en el libro acompañada de su "hermana" que representa $(x+2)^{2}=x^{2}+4 x+4$. No cabe duda que estas figuras tienen un carácter ilustrativo evidente. Sin embargo, este tipo de representaciones tiene dos defectos: da al producto una naturaleza de área, distinta de la naturaleza de longitud que los factores tienen, y puede contribuir a reforzar la idea, frecuente en las producciones de los estudiantes aun cuando no la explicitan, que la variable $x$, cuya escritura no enseña ningún signo "_“, toma solo valores positivos. Lo cual es fuente de muchos errores de signo señalados en reportes de investigación. Por ejemplo Cortes y Kavafian (1999) señalan errores cometidos por estudiantes en la resolución de la ecuación $-9 y=99$, que ellos transforman frecuentemente en $y=\frac{99}{9}$ o en $-y=\frac{99}{-9}$, lo que les conduce a dar como "solución" $y=11$ (positiva) en vez de $y$ $=-11$ (negativa).

Un hecho es que el enunciado de la regla de los signos es muy operativo, lo que es suficiente para los usos inmediatos en problemas de contabilidad y problemas ulteriores, como son la resolución de muchas ecuaciones sencillas y el estudio de variaciones de funciones. Además, el uso de herramientas digitales facilita las operaciones y reduce los riesgos de errores. Una causa posible es que a los responsables de decisiones a nivel del sistema educativo no les parezca necesario invertir mucho tiempo de enseñanza en la comprensión de la "regla de los signos". Sin embargo, pensamos que las consecuencias de la incomprensión de la justificación de la regla de los signos no son mínimas. Y nuestra 
hipótesis es que es posible mejorar su enseñanza bajo la condición de introducir un enfoque más completo, el cual vamos a presentar a continuación.

\section{Una construcción de Descartes en el marco de los espacios de trabajo matemático}

\subsection{Presentación de la construcción de Descartes}

Un diagnóstico que resulta de las consideraciones anteriores se puede precisar a la luz de los Espacios de Trabajo Matemático (ETM, KUZNIAK; RICHARD, 2014). En el esquema de génesis entre un plano cognitivo y un plano epistemológico propuesto por Kuzniak y Richard (ibid. Figura 2, p. 9) aparecen: una génesis semiótica entre visualización y representamen, una génesis instrumental entre construcción y artefactos, una génesis discursiva entre prueba y referencial. En nuestro caso se puede considerar que la génesis instrumental está favorecida por el fuerte énfasis que hay sobre las operaciones y los algoritmos operatorios (ejemplo de la regla de los signos), mientras cierta carencia existe respecto de la génesis discursiva (ejemplo del estatuto de la distributividad del producto sobre la suma que no se explicita) y sobre todo que casi no se considera la génesis semiótica, en ausencia de representación sensible del producto de enteros y más generalmente de productos en los que se pueden presentar números negativos.

En los planes y programas de estudio, ¿dónde encontrar un recurso posible para la representación del producto de enteros? En el volumen 2 del libro de la SEP citado, un bloque geométrico (Secuencia 16, p. 36) presenta una situación, el teorema de Tales, en la que aparecen razones y proporciones a propósito de rectas paralelas. ¿Se puede entonces encontrar en esta situación una representación adecuada del producto? 

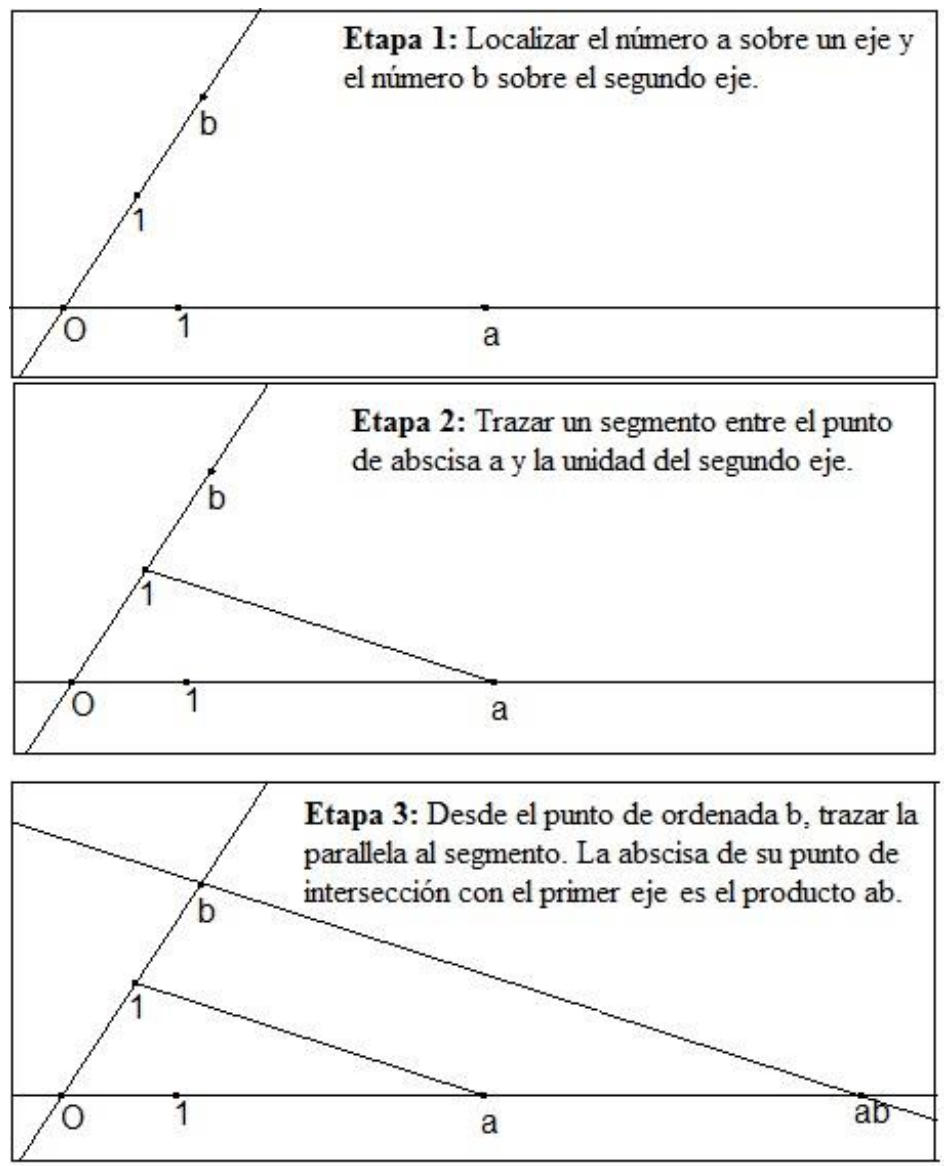

Figura 8 - Producto ab obtenido por una construcción de Descartes.

Precisamente, una construcción de Descartes (1637) parece cumplir todos los requisitos para dar una buena interpretación geométrica del producto de dos números $a$ y $b$ sin o con signos (en este caso, sólo hace falta prolongar las rectas del lado negativo). La ilustramos en la Figura 8. En particular, el producto $a b$ tiene la misma naturaleza de longitud que cada uno de sus factores tiene. Por consecuencia, el resultado no plantea los problemas de magnitudes que ocurren con un producto de longitudes visto como un área.

\subsection{Estudio empírico}

Para una enseñanza que tomaría en cuenta los elementos precedentemente descritos, nos dirigimos en primer lugar al público de estudiantes para profesor de matemáticas. Se aplicó un cuestionario en el que se presentó la construcción de Descartes dentro del marco de un estudio comparado de la formación inicial entre Francia (un grupo de estudiantes de la universidad de Paris-Diderot) y México (un grupo de estudiantes de la Escuela Normal Superior del Estado de Morelos). Los resultados observados se publicaron en un artículo de 
Juárez, Arredondo \& Pluvinage (2014). En este estudio la construcción de Descartes dio lugar a una pregunta que resultó fácil para el grupo francés y difícil para el grupo mexicano. Por esta razón nos interesó confirmar la observación con base en un cuestionario aplicado en 2015 a una pequeña muestra de futuros profesores en la Ciudad de México. ${ }^{2}$

El cuestionario se aplicó a 9 estudiantes entre 19 y 23 años al término del cuarto semestre de la licenciatura para profesor de Matemáticas de educación secundaria en la Normal Superior de México, ubicada en la Ciudad de México.

En el cuarto semestre los estudiantes ya han cursado las siguientes asignaturas:

- Conceptos matemáticos: Figuras y cuerpos geométricos; Plano cartesiano y funciones; Procesos de cambio o variación.

- Psicología del adolescente: Desarrollo de los adolescentes IV; Procesos cognitivos.

- Pedagogía y didáctica: Seminario de temas selectos de historia de la pedagogía y la educación I; Planeación de la enseñanza y evaluación del aprendizaje; Observación y práctica docente II.

En la asignatura plano cartesiano y funciones los estudiantes relacionan expresiones algebraicas con sus respectivas representaciones gráficas. Los contenidos estudiados en esta asignatura, así como los de semestres anteriores, podrían ser herramientas útiles para que los estudiantes respondieran el cuestionario.

Sin embargo los estudiantes declararon no haber estudiado aun lo referido a Descartes y con este argumento no contestaron la parte referida a la obtención geométrica del producto (véase la pregunta M2 en el Anexo).

Por otro lado, cabe la posibilidad de que hayan considerado el tema muy complejo al ligarlo con la filosofía, con la que asocian a Descartes, y sentir la necesidad de leer con antelación lo desarrollado por Descartes ya que argumentaron 'Apenas lo vamos a estudiar'; y repitieron el mismo argumento cuando se les propuso utilizar el teorema de Tales.

También se observa que al estudiar los contenidos se deja pasar por alto la visualización gráfica como una parte didáctica importante con la que toman sentido algunos conceptos y que puede constituir la base de una enseñanza que no pondere el álgebra sobre la geometría.

Con respecto a la solución de la ecuación con una raíz, los estudiantes mostraron respuestas diversas. Sin embargo, dentro de la diversidad denotan una ponderación de la aritmética sobre el álgebra y también la falta de cuidado y precisión en sus respuestas. En

\footnotetext{
${ }^{2}$ Se anexa la versión en español del cuestionario.
} 
particular, en relación con los problemas de signos, no señalaron que $A^{2}=B^{2}$ no implica $A=$ B. En efecto, en la respuesta dada por un estudiante de la Preparatoria, la igualdad

$\sqrt{7-x}=x-5$ es transformada en $7-x=(x-5)^{2}$ por elevación al cuadrado, sin ninguna explicación. Pero una solución de la segunda ecuación puede dar lugar a la igualdad $\sqrt{7-x}=-x+5$.

De los nueve estudiantes sólo uno hace referencia a que una de las soluciones a la ecuación se considera extraña porque no es la solución a la ecuación, pero no se propone buscar otras respuestas posibles y además ninguno de los estudiantes expresa esta posibilidad, sólo se concretan a las respuestas escritas.

En general los estudiantes no consideran la regla de los signos como una dificultad en la respuesta analizada, lo cual es relevante en tanto no hay una reflexión sobre el proceso de solución sino sólo una mecanización de la misma, lo cual implica un obstáculo en los procedimientos didácticos.

Cabe destacar que los estudiantes no señalaron dificultades en el tratamiento didáctico del tema tratado.

\section{3 ¿Es difícil la construcción de Descartes?}

Regresamos a la obtención de un producto por la construcción de Descartes: Hace falta aclarar por qué el análisis de la figura trazada se puede considerar como obvio por ciertos individuos e inalcanzable por otros.

Hipótesis: La geometría en la educación matemática mexicana se sitúa principalmente al nivel de Geometría I definido en la taxonomía de Houdement y Kuzniak (2006). Sin embargo, la aprensión de la construcción de Descartes ya necesita una deconstrucción (Duval, 2005) que sobrepasa este nivel.

En el artículo de Duval (2005) se enseña la figura siguiente (Figura 9), muy sencilla, y que a pesar de esta sencillez genera dificultades a estudiantes de la secundaria, para que ellos entiendan que esta situación constituye un contra-ejemplo del enunciado tentativo "dos rectángulos de misma área tienen el mismo perímetro". 


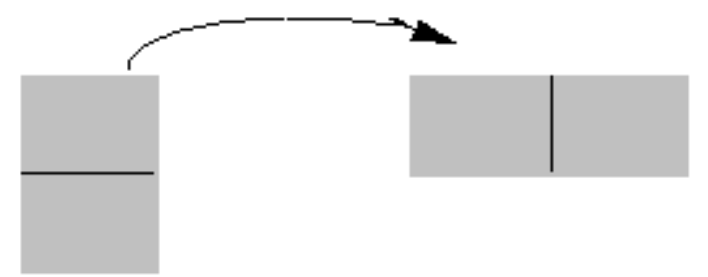

Figura 9 - tomada de Duval (2005, p. 35)

También podríamos ver esta figura como una ilustración de la ley asociativa $a \times(2 \times b)=(a \times 2) \times b$.

La figura de la construcción de Descartes se debe percibir no como un triángulo grande en el que se traza un segmento entre dos lados, sino como un caso de situación de Tales, con dos rectas secantes cortadas por dos paralelas. Dicho de otra manera, el aprendizaje debe de producir un efecto de cambio de percepción: En vez de la percepción espontanea, en la que las formas que se imponen son les contornos exteriores y les superficies elementales, se reconoce de inmediato "la situación de Tales". Y luego otra parte de la comprensión es la justificación de que el resultado obtenido es $a b$, porque es al número $a$ como el número $b$ es a 1. Este argumento supone un acceso al estrato proporcional descrito por Adjiage y Pluvinage (2012).

\section{Perspectivas: examen detallado de la relación entre números y geometría}

Bruno (2001) indica que, para reflexionar sobre los elementos del campo conceptual que rodean al concepto de número, se necesitan distinguir tres dimensiones del conocimiento numérico: 1) la dimensión abstracta; 2) la dimensión de recta; 3) la dimensión contextual. En el caso de los números negativos, hemos visto que la dimensión abstracta necesita la distributividad del producto sobre la suma, conforme a lo que Glaeser (1981) acierta a propósito de la construcción de los enteros. Por tanto esta propiedad tiene un estatuto bastante sutil:

- En el caso de números positivos (e. g. los naturales), la distributividad es un teorema.

- Para la extensión a negativos, la distributividad se debe enunciar como axioma.

1) La extensión de las prácticas es la parte medular de estudios que abarcan los números que nos interesan aquí, así como tópicos más avanzados, por ejemplo el álgebra en Gallardo (2002), los números complejos en Schneider, Job, Matheron y Mercier (2015). 
2) La dimensión geométrica de la recta, es decir la dimensión espacial 1, es suficiente en contextos aditivos. Pero los contextos multiplicativos obligan a desarrollar un proceso bidimensional. Sin embargo, este no debe conducir a cambiar de magnitudes.

3) La dimensión contextual del producto aparece en diversas situaciones, como los momentos de fuerzas en situaciones físicas (palancas o balanzas de dos platillos), o la posición en un instante dado (pasado o futuro) de un móvil animado de un movimiento uniforme, e. g. un tren sobre un carril rectilíneo.

Debido al segundo punto, se necesita un proceso geométrico cuyo resultado sea el producto de dos números representados como puntos de rectas graduadas. El proceso que experimentamos se define a partir de la situación del teorema de Tales, y el experimento sugiere que el dominio de un mero proceso supone una cultura matemática relativamente avanzada. Sin embargo, una visión proceptual (Sfard, 1991 y Gray y Tall, 1994) abre otras perspectivas de aprendizaje. Sfard (1991) consideró proceso y objeto como los dos lados de una misma moneda, y Gray y Tall (1994) introdujeron la palabra procept como la combinación de proceso y objeto. En la geometría, las transformaciones del plano tienen esta doble naturaleza. En particular en nuestro caso, la homotecia introduce de manera geométrica el producto por un factor, llamado la razón de la homotecia. La imagen de un segmento $s$ por una homotecia es un segmento s' paralelo. A la figura obtenida (véase Figura 10) se aplica el teorema de Tales.

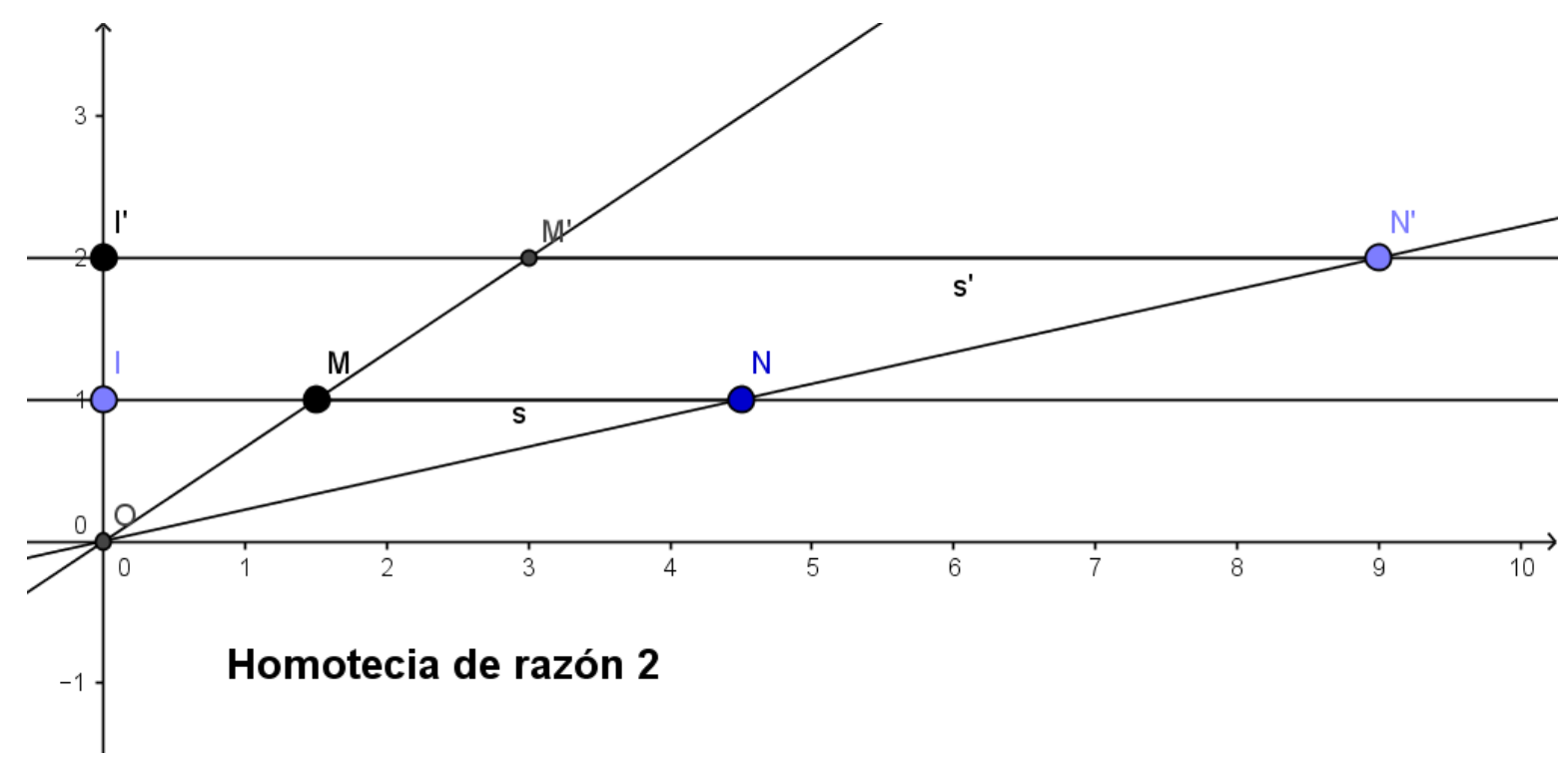

Figura 10 - Imagen de un segmento por una homotecia de centro O.

¿Por qué no propusimos la homotecia en nuestro cuestionario? La explicación es que en el libro de la SEP como en los otros libros de texto del mismo nivel, el teorema de Tales se considera antes de la homotecia, y que en los planes y programas de 2011 (SEP, 2011), 
actualmente vigentes, no se menciona la homotecia. Entonces experimentamos con los estudiantes para maestros de matemáticas un cuestionario cuya rúbrica sobre el producto de enteros se apoya sobre la construcción de Descartes justificada por el teorema de Tales. Pero la observación sugiere que el entendimiento de esta construcción necesita una cultura matemática avanzada: Por ejemplo, los estudiantes para profesores franceses que contestaron de manera exitosa habían estudiado herramientas de geometría proyectiva como es la razón anarmónica (o razón doble). Además la prueba de la distributividad en la construcción de Descartes es compleja.

Al contrario, de la homotecia se deduce sencillamente la distributividad: De la invariancia observada en la Figura 10 resulta que las imágenes de dos segmentos pegados colineales son también segmentos pegados colineales. Por otro lado, el uso de esta transformación es muy común, hasta en pantallas táctiles (Figura 11).

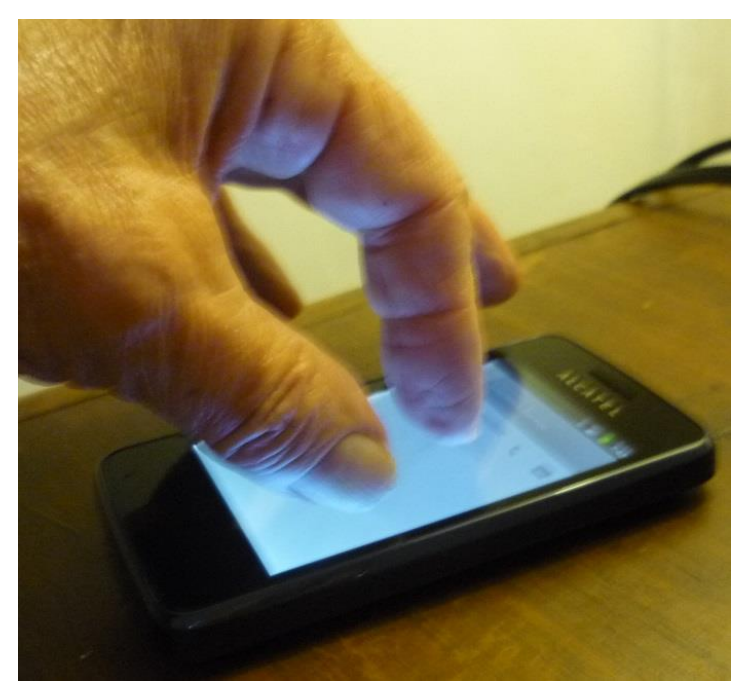

Figura 11 - Homotecia gestual en la pantalla de un smart-phone

Nuestra hipótesis entonces es que el aprendizaje de la regla de los signos se puede llevar a cabo con la representación geométrica del producto por la homotecia, como lo muestra la Figura 12 en el caso de un producto de dos números negativos. 


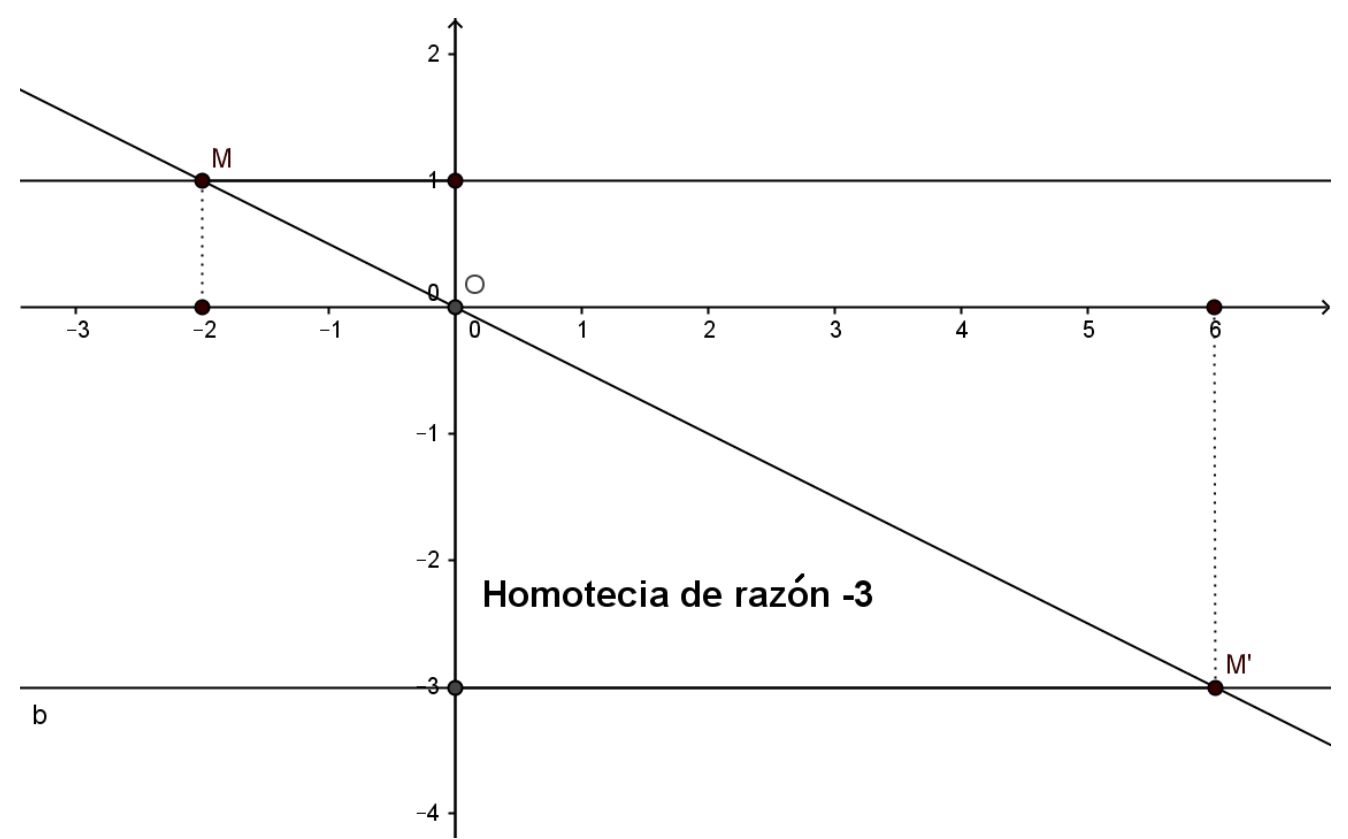

Figura 12 - Ilustración del producto $(-2) \times(-3)=6$

Nos interesará en el futuro comprobar esta hipótesis de factibilidad en un experimento con uso de software de geometría dinámica, en un ambiente de trabajo colaborativo con profesores, semejante a lo que ya hemos llevado a cabo en una comunidad de practica (PARADA; PLUVINAGE; SACRISTÁN, 2013).

\section{Referencias}

ADJIAGE, R; PLUVINAGE, F. Strates de compétences en mathématiques. Repères IREM, Nancy, v. 88, p.43-72, jui. 2012.

BRUNO, A. Algunas investigaciones sobre la enseñanza de los números negativos. In: SIMPOSIO DE LA SOCIEDAD ESPAÑOLA DE INVESTIGACIÓN EN EDUCACIÓN MATEMÁTICA, 4, 2001, Huelva. Actas, Huelva: Universidad de Huelva, 2001. p. 119 - 130.

BRUNO A; MARTINÓN A. Contextos y estructuras en el aprendizaje de los números negativos. Suma, v. 16, España. 1994. Disponible en: 〈http://revistasuma.es/IMG/pdf/16/009-018.pdf〉. Acceso: 8 mar. 2016.

CAJORI, F. History of Mathematical Notations. Chicago: The Open Court publishing $\mathrm{C}^{\circ}$ (Chicago University press), 1928-29. v. 1-2.

CID, E. Obstáculos epistemológicos en la enseñanza de los números negativos. Actas de las XV Jornadas del Seminario Interuniversitario de Investigación en Didáctica de las Matemáticas, Boletín del SI-IDM, 10. 2000. Disponible en : 〈http://www.ugr.es/ jgodino/siidm/cangas/Negativos.pdf $>$. Acceso: 8 mar.2016.

CORTES, A; KAVAFIAN, N. Les principes qui guident la pensée dans la résolution des équations. Petit x, Grenoble, v. 51, p. 47-73, jui. 1999. 
DESCARTES, R. Discours de la méthode. Leyde, 1637. Disponible en:

$<$ http://www.bibnum.education.fr/math\%C3\%A9matiques/g\%C3\%A9om\%C3\%A9trie/le-livrepremier-de-la-g\%C3\%A9om\%C3\%A9trie-de-descartes>. Acceso: 8 mar. 2016.

DUVAL, R. Les conditions cognitives de l'apprentissage de la géométrie : développement de la visualisation, différenciation des raisonnements et coordination de leurs fonctionnements. Annales de Didactique et de Sciences Cognitives. Strasbourg, v. 10, p. 5 53, 2005.

GALLARDO, A. The extension of the natural-number domain to the integers in the transition from arithmetic to algebra. Educational Studies in Mathematics, Netherlands, v. 49, n. 2, p. 171-192, feb. 2002.

GLAESER G. Epistémologie des nombres relatifs. Recherches en Didactique des Mathématiques, Grenoble, v. 2, n. 3, p. 303-346.1981.

GRAY, E.; TALL, D. Duality, ambiguity, and flexibility: A "proceptual" view of simple arithmetic. Journal for Research in Mathematics Education, Reston, v. 25, n. 2, p. 116 - 140, mar. 1994.

HOUDEMENT, C.; KUZNIAK, A. Paradigmes géométriques et enseignement de la géométrie. Annales de Didactique et de Sciences Cognitives, Strasbourg, v.11, p. 175 - 193. 2006.

JUÁREZ, M.; ARREDONDO, A. ; PLUVINAGE, F. Etude comparée de la formation initiale de professeurs de mathématiques en France et au Mexique. Annales de Didactique et de Sciences Cognitives, Strasbourg, v.19, p. 251- 283. 2014.

KUZNIAK, A. ; RICHARD. Espacios de trabajo matemático. Puntos de vista y perspectivas. Revista Latinoamericana de Investigación en Matemática Educativa, México, v.17, n. 4-I, p. 5 - 15. 2014.

JUÁREZ, M.; ARREDONDO, A.; PLUVINAGE, F. Etude comparée de la formation initiale de professeurs de mathématiques en France et au Mexique. Annales de Didactique et de Sciences Cognitives, Strasbourg, v.19, p. $251-283.2014$.

MAZ, A.; RICO, L. Situaciones asociadas a los números negativos en textos de matemáticas españoles de los siglos XVIII y XIX. España, 2007. Disponible en:

<http://www.pna.es/Numeros2/pdf/Maz2007PNA1\%283\%29Situaciones.pdf > Acceso: 8 mar. 2016.

PARADA, S.; PLUVINAGE, F.; SACRISTÁN A. Reflexiones en una comunidad de práctica de educadores matemáticos sobre los números negativos, Recherches en Didactique des Mathématiques, Grenoble, v.33, n.3, p. 233 -267. 2013.

SCHNEIDER, M. et al.. Extensions praxémiques liées aux ensembles de nombres: des complexes aux relatifs. Annales de Didactique et de Sciences Cognitives. Strasbourg, v.20, p. 9-46. 2015.

SFARD, A. On the dual nature of mathematical conceptions: Reflections on processes and objects as different sides of the same coin. Educational Studies in Mathematics, Netherlands, v. 22, n. 1, p. 1 36, feb. 1991.

STEVIN, S. L'arithmétique. Free Google eBook . 1585. Disponible en: $<$ http://books.google.fr/books/about/L arithmetique.html?hl=fr\&id=1dU5AAAAcAAJ > Acceso: 8 mar. 2016.

VIÈTE, F. In artem analyticem isagoge. 1591). Disponible en: $<$ http://gallica.bnf.fr/ark:/12148/bpt6k108865t.r=Fran\%C3\%A7ois+Vi\%C3\%A8te.langFR > Acceso: 8 mar. 2016. 


\section{Documentos oficiales}

MÉXICO. Secretaría de Educación Pública. Telesecundaria, 3er grado, Matemáticas. Libro de texto gratuito de dos volúmenes. México: SEP, 2009.

MÉXICO. Secretaría de Educación Pública Programas de estudio, Guía para el maestro, Educación básica secundaria, Matemáticas. México, 2011. Disponible en:

<http://basica.sep.gob.mx/dgdc/sitio/pdf/inicio/matlinea/2011/Matematicas SEC.pdf $>$ Acceso: 8 mar. 2016.

Submetido em Julho de 2015. Aprovado em Setembro de 2015. 


\section{Anexo}

\section{Cuestionario adaptado de Juárez, Arredondo y Pluvinage (2014)}

Este cuestionario no da lugar a una evaluación individual, le agradecemos de antemano su colaboración.

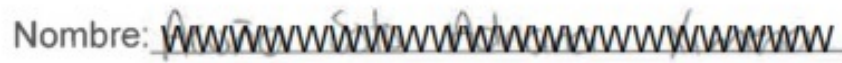

\section{CUESTIONARIO DE MATEMÁTICAS}

Recomendación: Usar calculadora programable o computadora personal.

M1. En el campo R de los números reales se considera la siguiente ecuación dependiente del parámetro a:

$$
x+\sqrt{x^{2}-4 a x}=3 a
$$

Ponga una cruz en la celda conveniente de cada una de las columnas de la tabla de abajo.

En el caso

\begin{tabular}{|c|c|c|c|}
\hline$a<0$ & $a=0$ & $a>0$ & \\
\hline \multirow[t]{3}{*}{ X } & & & la ecuación no tiene solución \\
\hline & $x$ & & la ecuación tiene 1 solución \\
\hline & & $x$ & la ecuación tiene 2 soluciones \\
\hline \multirow[t]{2}{*}{$x$} & & & la ecuación tiene una infinitud de soluciones que no cubre $R$ \\
\hline & $x$ & & La ecuación tiene todo elemento de $\mathrm{R}$ como solución \\
\hline
\end{tabular}

Para contestar, ¿usó usted una herramienta de cómputo? SI $\square \quad$ No $\square$

En caso de que tu respuesta sea afirmativa favor de precisar la herramienta y su uso: 
M2. A partir de dos rectas graduadas de mismo origen en el plano, el filósofo y matemático René Descartes propone en su Discours de la Méthode un método geométrico para la multiplicación de dos números, que sean positivos o negativos.

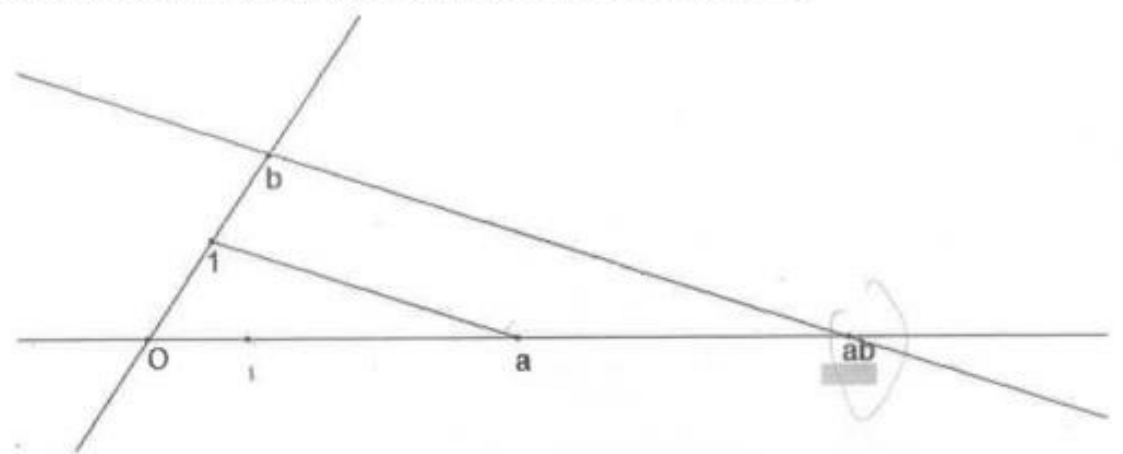

Figura 1. Obtención geométrica del producto ab por el método de Descartes.

La Figura 1 ilustra el método de Descartes en el caso de dos números positivos.

1) Al igual de Descartes, describa la construcción ilustrada para obtener geométricamente

Lo el producto ab. la ... xegla de congresencia.

2) Justifique que se obtiene ab con esta construcción.

3) La figura trazada ilustra el método de Descartes en al caso de dos números positivos. A la luz de la construcción, comente la regla de los signos para el producto de números

$$
{ }_{(-)}^{\text {negativos. }}(-)=t(-)(t)=-(t)(-)=-
$$

4) Ilustre geométricamente la distributividad del producto sobre la suma.

M3. ¿Cuál es el lugar geométrico del ortocentro de un triángulo $A B C$ cuyos vértices $A$ y $B$ son fijos, mientras $C$ se mueve sobre una paralela dada a $A B$ ? Justifica tu respuesta.

Recomendación: En un sistema de ejes escogido a propósito, utilice geometría analitica. 
Algunas cuestiones sobre didáctica de las matemáticas

D1 ¿Puede asociar algún concepto didáctico a cada uno de los siguientes nombres?

Sócrates: Números y relaciones.

Piaget: conductismo y razoncimiento.

vygotsky: Etapas del adolescente y el censamiento matemitico ¿Qué didacta de las matemáticas conoce? el razona mi ento del porque?

D2 Pon una calificación de 0 a 5 (como máximo) a la resolución siguiente de ecuación por un estudiante de tercer año de secundaria, y propón un comentario dirigido al mismo estudiante.

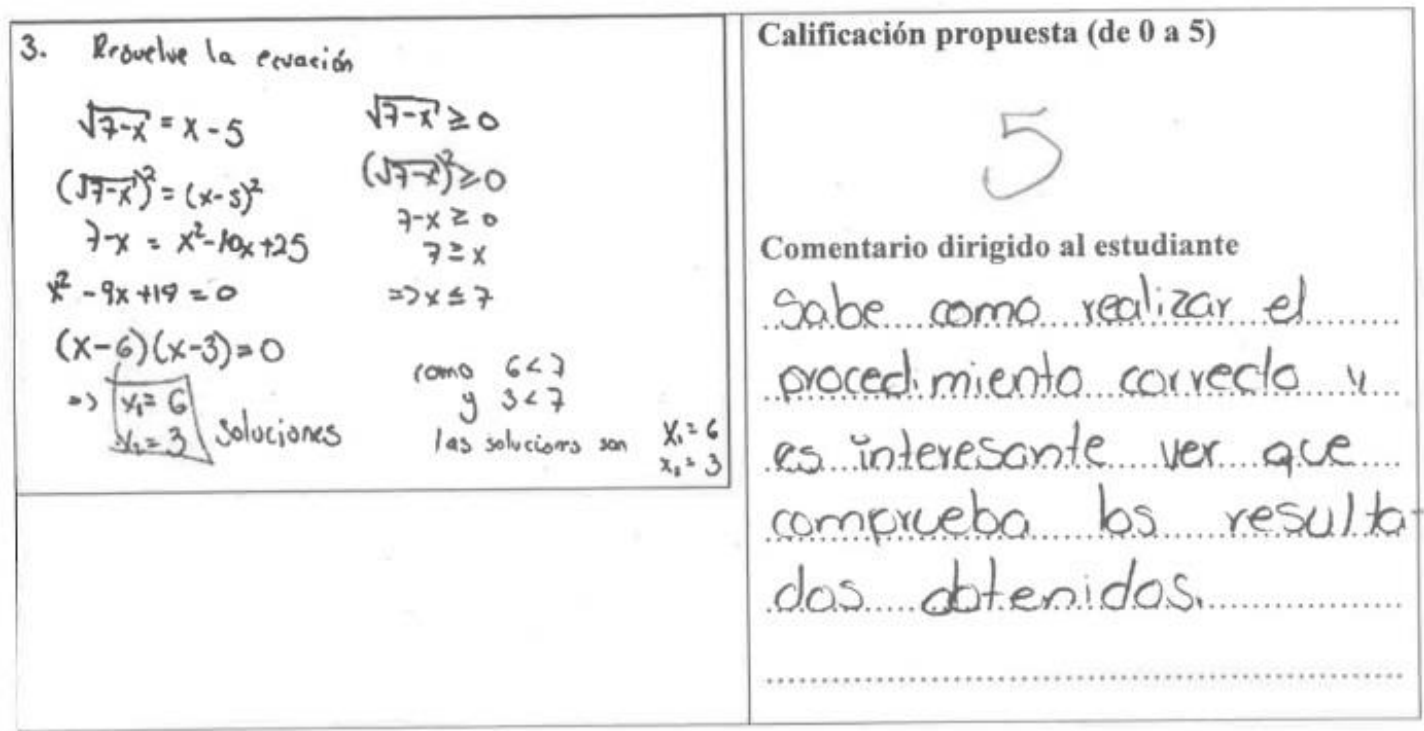

Llegó al fin del cuestionario, gracias por su colaboración 